\title{
Lack of Control by Glucose of Ultradian Insulin Secretory Oscillations in Impaired Glucose Tolerance and in Non-insulin-dependent Diabetes Mellitus
}

Niall M. O'Meara, Jeppe Sturis, Eve Van Cauter, and Kenneth S. Polonsky

The Department of Medicine, University of Chicago, Pritzker School of Medicine, Chicago, Illinois 60637

\begin{abstract}
Normal subjects demonstrate the presence of ultradian oscillations (period 80-150 $\mathrm{min}$ ) in insulin secretion rate (ISR) tightly coupled to glucose oscillations of similar period. These oscillations appear to be a function of the feedback loop linking glucose and insulin. The present study was undertaken to determine whether the control by glucose of the ultradian oscillations in insulin secretion is altered in impaired glucose tolerance IGT and in non-insulin-dependent diabetes mellitus (NIDDM). Patients with NIDDM $(n=7)$, IGT $(n=4)$, and matched nondiabetic controls $(n=5)$ were studied under three separate protocols that involved administration of glucose at either a constant rate of $6 \mathrm{mg} / \mathrm{kg}$ per min for $28 \mathrm{~h}$ or in one of two oscillatory patterns at the same overall mean rate. The amplitude of the oscillations was $33 \%$ above and below the mean infusion rate, and their respective periods were $144 \mathrm{~min}$ (slow oscillatory infusion) or $96 \mathrm{~min}$ (rapid oscillatory infusion). Insulin, C-peptide, and glucose were sampled at 10 -min intervals during the last $24 \mathrm{~h}$ of each study. ISRs were calculated by deconvolution of C-peptide levels. Analysis of the data showed that $(a)$ the tight temporal coupling between glucose and ISR in the nondiabetic controls was impaired in the IGT and NIDDM groups as demonstrated by pulse analysis, crosscorrelation analysis, and spectral analysis; $(b)$ the absolute amplitude of the ISR pulses progressively declined with the transition from obesity to IGT to NIDDM; and (c) the absolute amplitude of the ISR oscillations failed to increase appropriately with increasing absolute amplitude of glucose oscillations in the IGT and NIDDM subjects compared with the control group. In conclusion, the present study demonstrates that important dynamic properties of the feedback loop linking insulin secretion and glucose are disrupted not only in established NIDDM but also in conditions where glucose tolerance is only minimally impaired. Further studies are needed to determine how early in the course of beta-cell dysfunction this lack of control by glucose of the ultradian oscillations in insulin secretion occurs and to define more precisely if this phenomenon plays a pathogenetic role in the onset of hyperglycemia in genetically susceptible individuals. ( $J$. Clin. Invest. 1993. 92:262-271.) Key words: entrainment • feedback loop • glucose • insulin secretion • oscillations $\bullet$ impaired glucose tolerance $\cdot$ non-insulin-dependent diabetes mellitus
\end{abstract}

Address reprint requests to Kenneth S. Polonsky, M.D., University of Chicago, Department of Medicine, MC1027, 5841 South Maryland Avenue, Chicago, IL 60637.

Received for publication 9 September 1992 and in revised form 3 February 1993.

J. Clin. Invest.

(C) The American Society for Clinical Investigation, Inc.

$0021-9738 / 93 / 07 / 262 / 10 \quad \$ 2.00$

Volume 92, July 1993, 262-271

\section{Introduction}

During the past 10-15 years, many studies have demonstrated that insulin secretion is a complex oscillatory process characterized by rapid oscillations with a periodicity of 8-15 min, which are superimposed on slower (ultradian) oscillations with a period of 1.5-2 $\mathrm{h}$ (1-9). Both rapid and slow oscillations have been observed under fasting conditions and during constant glucose infusion. The ultradian oscillations have in addition been demonstrated during constant enteral nutrition and following meals. The rapid pulses persist in the isolated perfused pancreas (10) and in isolated islets (11) exposed to a constant glucose concentration, suggesting that they occur independently of oscillations in plasma glucose and that they originate due to intraislet mechanisms. The ultradian oscillations in insulin secretion are, by contrast, tightly coupled to glucose (6, $8,9)$. Studies in normal weight subjects during constant glucose infusion and following meals have demonstrated a high degree of concomitance between these secretory oscillations and oscillations in glucose of comparable periodicity. In addition, the periodicity of the insulin secretory oscillations can be entrained to the period of an oscillatory glucose infusion (9). These findings are entirely compatible with the view that the ultradian oscillations in insulin secretion are a product of the insulin/glucose feedback mechanism $(12,13)$.

We have studied the temporal interactions between glucose and insulin secretion by determining the extent to which ultradian oscillations in insulin secretion can be entrained by exogenous glucose. The concept of entrainment is central to the field of nonlinear dynamics and has been used in many different scientific fields, including the study of biological systems (14). Briefly, if a nonlinear, self-oscillating system is perturbed exogenously with a periodic stimulus, different types of oscillatory behaviors may emerge, one of which is entrainment. When entrainment occurs, the oscillation of the system reacts to the exogenous stimulus by adjusting its period to that of the stimulus. The ability to entrain depends on a number of factors, including the period of the stimulus compared with the natural period of the system, the amplitude of the stimulus, the nonlinearities of the system, and the strength of the coupling between the exogenous stimulus and the system.

In non-insulin-dependent diabetes mellitus (NIDDM), ${ }^{1}$ the oscillatory pattern of $\beta$-cell secretion is disrupted. The persistent, regular rapid oscillations present in normal subjects are replaced by irregular cycles of shorter duration both in subjects with established NIDDM and in their first-degree relatives (15, 16). The ultradian oscillations in NIDDM are also more irregular. In addition, they are of smaller amplitude and the proportion of these oscillations that are concomitant with oscillations

1. Abbreviations used in this paper: BMI, body mass index; IGT, impaired glucose tolerance; ISR, insulin secretion rate; NIDDM, non-insulin-dependent diabetes mellitus. 
in glucose is much smaller $(17,18)$. These latter observations raise the possibility that specific dynamic properties of the feedback loop linking glucose and insulin are disrupted in NIDDM. Such an alteration, if it could be confirmed, might be indicative of an important early $\beta$-cell abnormality in susceptible individuals even before the onset of overt hyperglycemia. The present study was therefore undertaken to determine if the ability to entrain $\beta$-cell secretion was reduced in subjects with established NIDDM and in patients with early impaired glucose tolerance (IGT) who had not yet developed overt hyperglycemia.

\section{Methods}

\section{Subjects}

Studies were performed in patients with proven NIDDM $(n=7,6$ males, 1 female; 2 Caucasian, 5 Black), impaired glucose tolerance ( $n$ = 4, 2 males, 2 females, 1 Caucasian, 3 Black), and in a group of obese subjects ( $n=5,4$ males, 1 female, 3 Caucasian, 1 Black, 1 Hispanic) who had normal glucose tolerance and no family history of diabetes. The criteria used to determine whether glucose tolerance was normal or impaired in the respective groups were those of the National Diabetes Data Group (19). The mean age, weight, and body mass index (BMI) of the three groups are demonstrated in Table I. None of the diabetic patients was receiving insulin therapy at the time of the study. Oral hypoglycemic agents were discontinued at least $2 \mathrm{wk}$ before the commencement of the experiments.

All studies were performed in the Clinical Research Center (CRC) of the University of Chicago. The protocol was approved by the Institutional Review Board, and all subjects gave written informed consent.

\section{Experimental protocol}

Subjects were admitted to the CRC after an overnight fast. After a subject was admitted, an intravenous catheter was inserted into both forearms, one for glucose infusion and one for blood sampling. Beginning at 0800 hours, glucose was infused intravenously for a period of 28 $\mathrm{h}$, during which time the subjects remained in the recumbent position. Each study consisted of an initial 4-h period (0800-1200 hours) to allow a steady state to be achieved following the transient glucose rise that is associated with the first few hours of initiating a glucose infusion (8). This was followed by a subsequent period of $24 \mathrm{~h}$ (1200-1200 hours) during which time samples were drawn at 10 -min intervals for glucose, insulin, and C-peptide. Lights were dimmed between 2300 and 0700 hours, to allow the subjects to sleep. Potassium ( $40 \mathrm{meq}$ ) was given orally every $12 \mathrm{~h}$, and subjects were allowed free access to water but not given any food for the duration of the experiment.

Each subject was studied on at least two occasions under the above protocol. On each occasion, glucose was administered as a $20 \%$ solution via a computer-controlled pump (Flo-gard 8000 volumetric infusion pump; Travenol Laboratories, Deerfield, IL) following different patterns. Initially, each subject received a constant glucose infusion at a rate of $6 \mathrm{mg} / \mathrm{kg}$ per min. The subsequent studies involved the administration of an oscillatory glucose infusion with periods of $96 \mathrm{~min}$ and/or $144 \mathrm{~min}$, these periods having been shown to be $20 \%$ shorter and $20 \%$ longer respectively than the endogenous period observed in normal subjects during constant glucose infusion (9). The software controlling the glucose infusion was custom written to allow the actual infusion rate to be recorded so that possible interruptions could be detected. Each oscillation was shaped as a sine wave and the amplitude was 33\% above and below the mean infusion rate. Subjects received identical volumes of glucose during each 28 -h infusion period.

\section{Analytical methods}

Blood samples for insulin measurements were allowed to clot at room temperature and the serum was stored at $-20^{\circ} \mathrm{C}$ until assayed. Samples for C-peptide were drawn into tubes at $4^{\circ} \mathrm{C}$ containing 500 Kallikrein inhibitor units $/ \mathrm{ml}$ Trasylol and $1.2 \mathrm{mg} / \mathrm{ml}$ EDTA. The plasma was immediately separated and stored frozen until assayed. Serum insulin was assayed by a double antibody technique (20). This assay has a lower limit of sensitivity of $20 \mathrm{pmol} /$ liter, and the average intraassay coefficient of variation is $8 \%$. Plasma C-peptide immunoreactivity was measured as previously described (21). The lower limit of sensitivity of the assay is $0.02 \mathrm{pmol} / \mathrm{ml}$ and the intraassay coefficient of variation averages $6 \%$. Plasma glucose was measured with a glucose analyzer (model 23A; Yellow Springs Instrument Co., Yellow Springs, $\mathrm{OH}$ ). The intraassay coefficient of variation of this method is $<3 \%$.

\section{Data analysis}

The relative degree of insulin resistance was evaluated with the Homeostasis Model Assessment (HOMA) method (22), using the mean of 3-4 stressed fasting glucose and insulin levels obtained on different occasions from each subject.

SMOOTHING AND ESTIMATION OF INSULIN SECRETION RATES The individual glucose, insulin, and C-peptide profiles were smoothed using a three-point moving average as in previous studies of oscillatory insulin secretion $(1,6,9)$. This procedure strongly dampens all fluctuations shorter than $30 \mathrm{~min}$, allowing a better visualization of slower oscillations at an expense of a modest reduction in their amplitude. It also reduces measurement error by a factor of $\sqrt{3}$. All further calculations were performed on the smoothed profiles. The smoothed C-peptide curve was used to derive insulin secretion rates by deconvolution (23). The kinetic parameters used in the deconvolution program were derived as previously validated and described in detail (24).

\section{PULSE ANALYSIS}

To identify significant pulses, each profile was analyzed with ULTRA, a computer program for pulse detection and quantification (25). The general principle of this algorithm is the elimination of all peaks for which either the increment (difference between the peak and the preceding trough) or the decrement (difference between the peak and the next trough) does not exceed a certain threshold related to measurement error. This produces a so-called "clean" profile. Extensive simulation studies (25) have indicated that a threshold of twice the intraassay coefficient of variation generally minimizes both false-positive and false-negative errors. This threshold was therefore used to identify significant pulses of glucose. However, because deconvolution involves an amplification of measurement error, a more conservative threshold of three times the intraassay coefficient of variation of C-peptide was used to quantify pulses of insulin secretion. In this study, pulse analysis was performed on the profiles smoothed by the three-point moving average so that the measurement errors were reduced by $\sqrt{3}$. Thus peaks of insulin secretion and glucose were considered significant if their respective increments and decrements exceeded $10.39 \%$ (i.e., 3 $\times 6 \% / \sqrt{3})$ and $3.46 \%(2 \times 3 \% / \sqrt{3})$, respectively. Peaks that did not meet these threshold criteria were eliminated from the data set using an iterative process. For each significant pulse, the absolute amplitude was defined as the difference between the level of the peak and the level of the preceding trough. Group statistics on pulse amplitudes were based on medians, rather than means, because of the non-Gaussian nature of pulse distribution.

Because glucose has a relatively long half-life, changes in production and/or utilization may not be reflected as significant peaks in the glucose concentration curve. We therefore identified all the "shoulders" in the glucose profiles. To do this, instantaneous derivatives of each individual clean glucose profile were estimated as the slope of the glucose changes during each sampling interval, and the shoulders were identified as slopes with an absolute value of zero (i.e., corresponding to the eliminated peaks), preceded and followed by slopes of the same sign.

ANALYSIS OF THE TEMPORAL ASSOCIATION BETWEEN OSCILLATIONS OF GLUCOSE AND OSCILLATIONS IN ISR Pulse-by-pulse analysis of concomitance. Temporal associations between oscillations in glucose and ISR were quantified by pulse-by-pulse 
analysis of the concomitance. Significant pulses of glucose and insulin secretion were considered concomitant if their peak values occurred within $10 \mathrm{~min}$ of each other. The concomitance ratio of glucose pulses with ISR pulses was calculated as the number of concomitant glucose and ISR pulses divided by the total number of glucose pulses. Conversely, the concomitance ratio of ISR pulses with glucose pulses was calculated as the number of concomitant glucose and ISR pulses divided by the total number of ISR pulses.

Cross-correlation analysis. For each glucose, insulin, and ISR profile a best-fit curve was calculated using a robust algorithm proposed by Cleveland (26) with a window of 15 data points. Each profile was then divided by its corresponding best-fit curve, thereby yielding a detrended profile for which diurnal variations were removed. For each study, coefficients of overall cross-correlation were then calculated between pairs of detrended profiles (glucose vs. insulin, glucose vs. ISR, and insulin vs. ISR) at lags of $0, \pm 10, \pm 20$, etc., up to $\pm 300 \mathrm{~min}$. Cross-correlation profiles from different studies were pooled using Fisher's $\mathrm{z}$ values (27). This procedure allowed the maximal coefficient of cross-correlation for each glucose infusion protocol and study group to be identified along with the lag at which this occurred, thus providing measures of the overall concomitance as well as the corresponding lag between the ultradian oscillations in glucose, insulin, and ISR.

Spectral analysis. To investigate the temporal nature of the profiles obtained during glucose infusion, spectral analysis was performed on each glucose, insulin, and ISR series. The methods are described by Jenkins and Watts (28). To remove circadian trends, each series was detrended with the first difference filter, and then spectral estimations were obtained using a Tukey window with a width of 72 data points. Each individual spectrum was normalized assuming the total variance of the series to be $100 \%$. This allowed average values across the three groups to be statistically compared.

STATISTICAL ANALYSIS

Results are expressed as mean \pm SEM. Statistical tests were performed using the Statistical Analysis System (version 6.04 for personal computers; SAS Institute Inc., Cary, NC). Repeated measures analysis of variance was used to identify overall differences between the three study groups and the studies using the three different glucose infusion protocols. Tukey's studentized range test was used for post-hoc comparisons. Differences were considered to be significant if $P<0.05$.

\section{Results}

Basal levels of plasma glucose, serum insulin, and ISR. The clinical characteristics and basal laboratory values of the study groups are recorded in Table I. All three groups were well matched for age, weight, and BMI. Fasting glucose and glycohemoglobin concentrations were significantly higher in the diabetic patients, while these values were normal in the patients

Table I. Ages, Weight, BMI, Basal Insulin, C-Peptide, Glucose, and $\mathrm{HbAl}$ in the Three Study Groups

\begin{tabular}{lccc}
\hline & Control & IGT & NIDDM \\
\hline & $(n=5)$ & $(n=4)$ & $(n=7)$ \\
Age (yr) & $40.4 \pm 4.6$ & $44.5 \pm 7.0$ & $46.0 \pm 4.7$ \\
Wt (kg) & $122 \pm 5.9$ & $110 \pm 11$ & $106 \pm 10$ \\
BMI (kg/m $\left.{ }^{2}\right)$ & $38.4 \pm 1.3$ & $38.8 \pm 4.4$ & $34.9 \pm 2.5$ \\
Insulin (pmol/liter) & $172 \pm 16.6$ & $128 \pm 4$ & $132 \pm 2.8$ \\
C-peptide (nmol/liter) & $1.06 \pm 0.13$ & $0.84 \pm 0.08$ & $0.87 \pm 0.13$ \\
Glucose (mmol/liter) & $5.54 \pm 0.21$ & $6.11 \pm 0.17$ & $10.3 \pm 0.98^{*}$ \\
Glycosylated hemoglobin (\%) & $5.81 \pm 0.18$ & $6.05 \pm 0.53$ & $10.3 \pm 1.1^{\dagger}$ \\
\hline
\end{tabular}

${ }^{*} P<0.001 ;{ }^{\dagger} P<0.01$ vs. control subjects. with IGT. Although basal glucose levels were slightly higher in those with IGT compared with the nondiabetic control subjects, the differences were not significant. The differences in the fasting insulin and C-peptide concentrations were not signifcant at the 5\% level. Use of the Homeostasis Model Assessment (HOMA) method revealed no significant differences in degree of insulin resistance between the three study groups (control, 8.0 \pm 1.4 ; IGT, $5.2 \pm 0.7$; NIDDM, 7.9 $\pm 1.4 ; P>0.43$ ).

Mean 24-h levels of plasma glucose, plasma C-peptide, serum insulin, and ISR. Overall mean concentrations of glucose, insulin, C-peptide, and the insulin secretory rates over the 24-h period during the constant glucose infusion studies are presented in Table II. In addition, the mean glucose levels and insulin secretory rates are shown for the oscillatory studies. Insulin, C-peptide levels, and insulin secretion rates were significantly lower in the NIDDM patients than in the nondiabetic controls, and the glucose concentrations in the NIDDM group were significantly higher than in both the control and IGT groups. The differences in glucose and insulin secretion rates between the patients with IGT and controls were not signifcant. The glucose levels and insulin secretion rates did not depend on the mode of glucose infusion. Fig. 1 depicts the mean insulin secretory rates and glucose levels during all studies. The mean glucose levels were elevated and insulin secretion rates were reduced in the subjects with NIDDM compared to controls, with virtually no overlap between the two groups. Patients with IGT tended to have low insulin secretion rates in relation to the prevailing glucose concentration, although the differences in secretion rate did not reach statistical significance.

Number and amplitude of oscillations. Representative profiles of glucose, insulin and ISR from each of the three study groups during the constant and oscillatory infusions are demonstrated in Figs. 2-4. The number and absolute amplitude of the respective oscillations in each experimental protocol are shown in Table III and Fig. 5. The number of glucose pulses was simi-

Table II. Mean Values ( \pm SEM) of Insulin, C-Peptide, Glucose, and Insulin Secretion during Constant Glucose Infusion and of Glucose and Insulin Secretion during Oscillatory Glucose Infusion

\begin{tabular}{|c|c|c|c|}
\hline & Control & IGT & NIDDM \\
\hline Constant glucose & $(n=5)$ & $(n=4)$ & $(n=7)$ \\
\hline Insulin (pmol/liter) & $1174 \pm 145$ & $591 \pm 101$ & $397 \pm 99^{*}$ \\
\hline C-peptide (nmol/liter) & $3.2 \pm 0.35$ & $2.4 \pm 0.23$ & $1.73 \pm 0.17^{*}$ \\
\hline Glucose (mmol/liter) & $9.2 \pm 0.47$ & $10.7 \pm 0.91$ & $20.4 \pm 1.83^{\dagger}$ \\
\hline $\begin{array}{l}\text { Insulin secretion } \\
\quad\left(\mathrm{pmol} / \mathrm{m}^{2} \text { per } \mathrm{min}\right)\end{array}$ & $426 \pm 48$ & $310 \pm 26$ & $229 \pm 23^{*}$ \\
\hline Oscillatory glucose-144 min & $(n=5)$ & $(n=3)$ & $(n=6)$ \\
\hline Glucose (mmol/liter) & $9.0 \pm 0.33$ & $11.4 \pm 0.74$ & $20.4 \pm 2.01$ \\
\hline $\begin{array}{l}\text { Insulin secretion } \\
\quad\left(\mathrm{pmol} / \mathrm{m}^{2} \text { per } \mathrm{min}\right)\end{array}$ & $416 \pm 31$ & $345 \pm 19$ & $242 \pm 26$ \\
\hline Oscillatory glucose- $96 \mathrm{~min}$ & $(n=5)$ & $(n=4)$ & $(n=5)$ \\
\hline Glucose (mmol/liter) & $9.4 \pm 0.52$ & $10.3 \pm 0.54$ & $19.1 \pm 1.91$ \\
\hline $\begin{array}{l}\text { Insulin secretion } \\
\left(\mathrm{pmol} / \mathrm{m}^{2} \text { per } \mathrm{min}\right)\end{array}$ & $416 \pm 37$ & $301 \pm 44$ & $243 \pm 30$ \\
\hline
\end{tabular}

${ }^{*} P<0.005$ vs. control; ${ }^{\dagger} P<0.0005$ vs. control and IGT. 


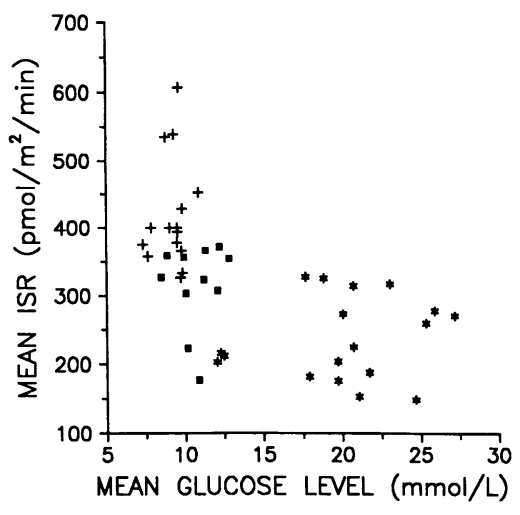

Figure 1. Relationship between mean insulin secretory rates normalized by body surface area and mean glucose concentrations during all glucose infusion studies. Each marking (control, +; IGT, $\mathbf{~}$, NIDDM, $\left.{ }^{*}\right)$ corresponds to the results of one study.

lar in the three groups except for a significant reduction during constant glucose infusion in the subjects with NIDDM $(P$ $<0.02$ ). The number of pulses of ISR did not differ in any of the three groups in any of the three protocols. Two-way analysis of variance for repeated measures showed that the amplitude of glucose oscillations increased during the oscillatory infusion studies, the changes being most marked with a slow 144-min oscillatory period $(P<0.0001)$. There were no signifcant differences in the amplitude of the glucose oscillations between the three groups. However, differences in the amplitude of the ISR oscillations were highly significant both between the groups $(P<0.001)$ and between the glucose infusion protocols $(P<0.01)$, and the group differences were related to the differences in the infusion protocols $(P<0.002)$. In the controls, the increase in amplitude of the glucose oscillations brought about by the oscillatory glucose infusion protocols was thus accompanied by a significant increase in the amplitude of the oscillations in ISR, whereas in both the diabetic and IGT groups, the amplitude of ISR oscillations did not increase significantly with the increase in amplitude of the glucose oscillations (Fig. 5).

Temporal association between oscillations in glucose and $I S R$. The temporal association between oscillations in glucose and ISR was evaluated using three separate approaches: pulse- by-pulse analysis of concomitance, cross-correlation analysis, and spectral analysis. Each approach to the analysis of the data indicated a reduction in the normal tight temporal coupling between oscillations in glucose and ISR in the NIDDM and IGT subjects although the significance of the differences varied depending on the technique.

Pulse-by-pulse analysis of concomitance. The concomitance between individual pulses of glucose and ISR for each group and protocol are shown in Table III. When the concomitance of pulses in ISR with glucose pulses was examined with repeated measures two-way analysis of variance, there was a significant difference between groups (NIDDM significantly different from IGT and control; $P<0.002)$ and between glucose infusion protocols $(P<0.0002)$. Similarly, the percentage of glucose pulses that was concomitant with an ISR pulse was significantly different between groups (NIDDM significantly different from control; $P<0.03$ ), but not between glucose infusion protocols. In order to determine if these differences were confirmed with other analytical approaches, cross-correlation and spectral analyses were applied.

Cross-correlation analysis. Fig. 6 illustrates the coefficient of cross-correlation between glucose and ISR at different lags for the three groups and the three glucose infusion protocols. Repeated measures two-way analysis of variance showed that the maximal coefficient of cross-correlation between glucose and ISR was different between the three groups (IGT and NIDDM different from control; $P<0.0001)$ and between the three glucose infusion protocols $(P<0.002)$. Similarly, the maximal coefficients of cross-correlation between glucose and insulin were different between the groups (IGT and NIDDM different from control; $P<0.001)$ and between the three glucose infusion protocols $(P<0.002)$. The results for correlation between ISR and insulin were only significant between the groups (NIDDM different from control; $P<0.04$ ). In order to examine which of the three glucose infusion protocols most clearly brought out group differences, one-way analysis of variance was performed on data from each glucose infusion study separately. The 144-min protocol consistently revealed the most significant differences. In addition to reduced overall
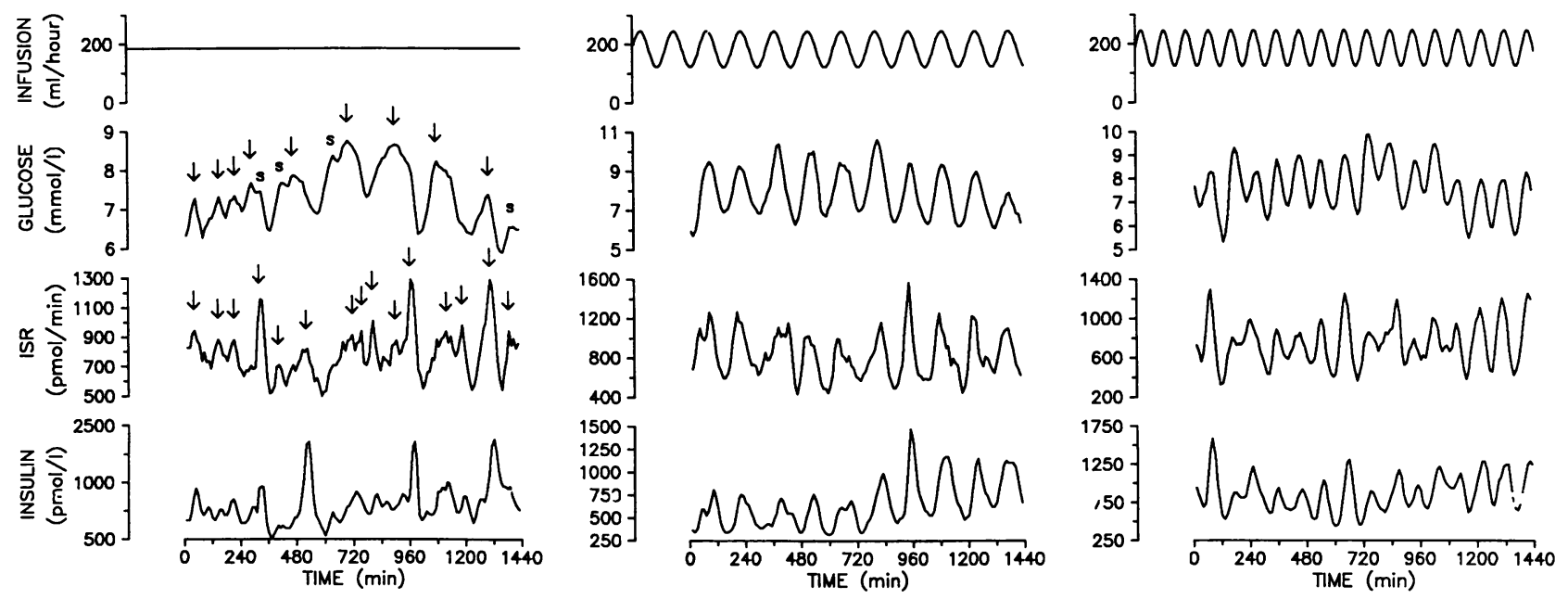

Figure 2. Profiles of the glucose infusion rate, the glucose and insulin concentrations, and the insulin secretory rates in one representative control subject. All three studies performed in this subject are shown: constant glucose infusion, left; slow oscillatory infusion, middle; rapid oscillatory infusion, right. Significant peaks are shown as arrows in the glucose and ISR profiles, and shoulder(s) are shown in the glucose profile obtained during constant glucose infusion. A few interruptions occurred that were all of short duration $(<2 \mathrm{~min})$, and they did not have any visible effect upon the glucose profiles. 

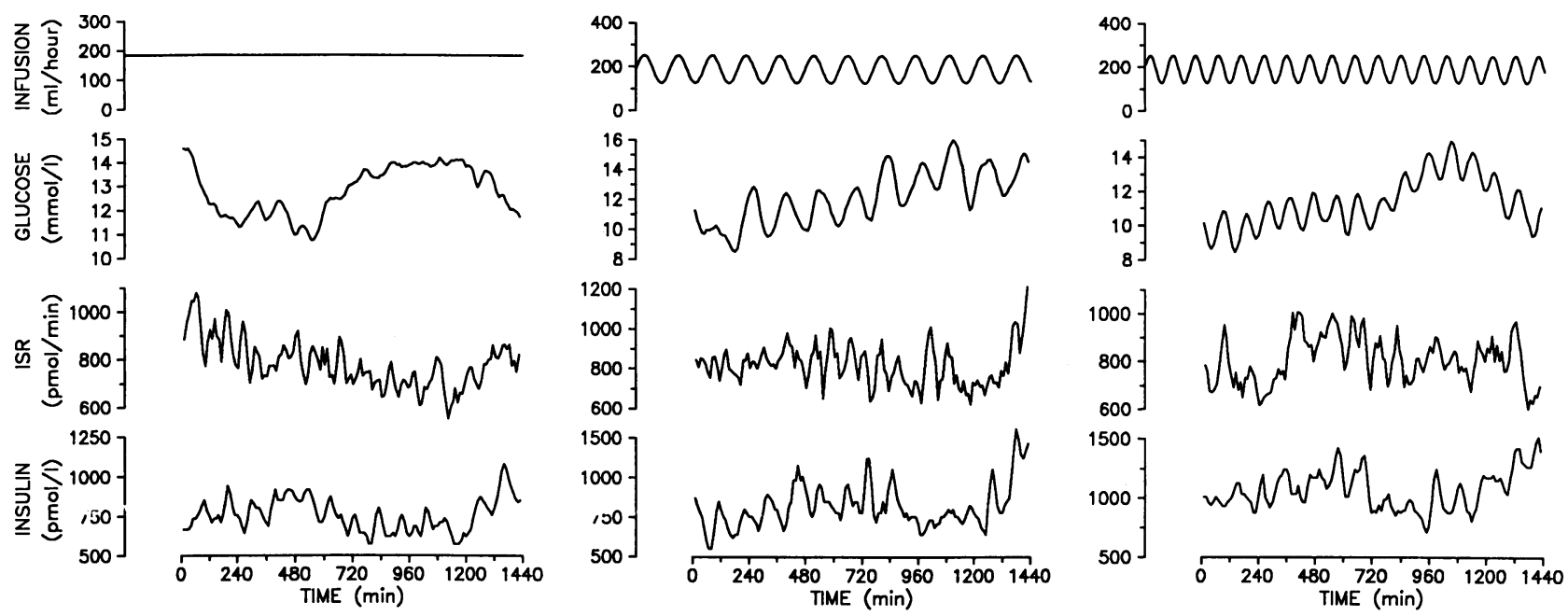

Figure 3. Profiles of the glucose infusion rate, the glucose and insulin concentrations, and the insulin secretory rates in one representative IGT patient. All three studies performed in this patient are shown: constant glucose infusion, left; slow oscillatory infusion, middle; rapid oscillatory infusion, right. A few interruptions occurred that were all of short duration $(<2 \mathrm{~min})$, and they did not have any visible effect upon the glucose profiles.

cross-correlation, the NIDDM group also demonstrated a considerably increased lag at which the maximal coefficient was observed (see Fig. 6). In other words, while the overall coefficient of cross-correlation between glucose and ISR was greatest at a lag of zero min in the control and IGT groups for all glucose infusion protocols, there was a lag in the NIDDM group which ranged from 15 to $35 \mathrm{~min}$ (peaks of glucose occurring before peaks of ISR) depending on the glucose infusion protocol. Similar results were obtained for the cross-correlation between glucose and insulin.

Spectral analysis. Spectral analysis of the oscillatory profiles (Fig. 7) confirmed the existence of peaks in the plasma glucose spectra in the three study groups at 96 and 144 min, corresponding to the respective periods of the exogenous infusion. Similar spectral peaks in insulin (not shown) and ISR were observed in the nondiabetic control subjects. However the power of the insulin and ISR spectral peaks was reduced in both the IGT and NIDDM groups, this reduction being most marked in the NIDDM group. During the 96-min oscillatory infusion the normalized power of the ISR spectrum fell from 29.1 in the controls to 13.6 in the subjects with IGT ( $P$ $<0.001)$ and $5.5(P<0.001)$ in the subjects with NIDDM. Correspondingly, the ISR spectra based on the 144-min oscillatory infusions yielded respective powers of 26.2, 5.9, and 3.0 in each of the three groups $(P<0.001)$. Similar results were obtained for the insulin spectra. No clear group differences were detected between the spectra from the constant glucose infusion protocol. Examination of individual spectra also showed that in a large proportion of the IGT (three of four) and NIDDM (five of seven) patients, a discrepancy between the dominant period of ISR and insulin could be detected. In contrast, none of the five nondiabetic control subjects exhibited
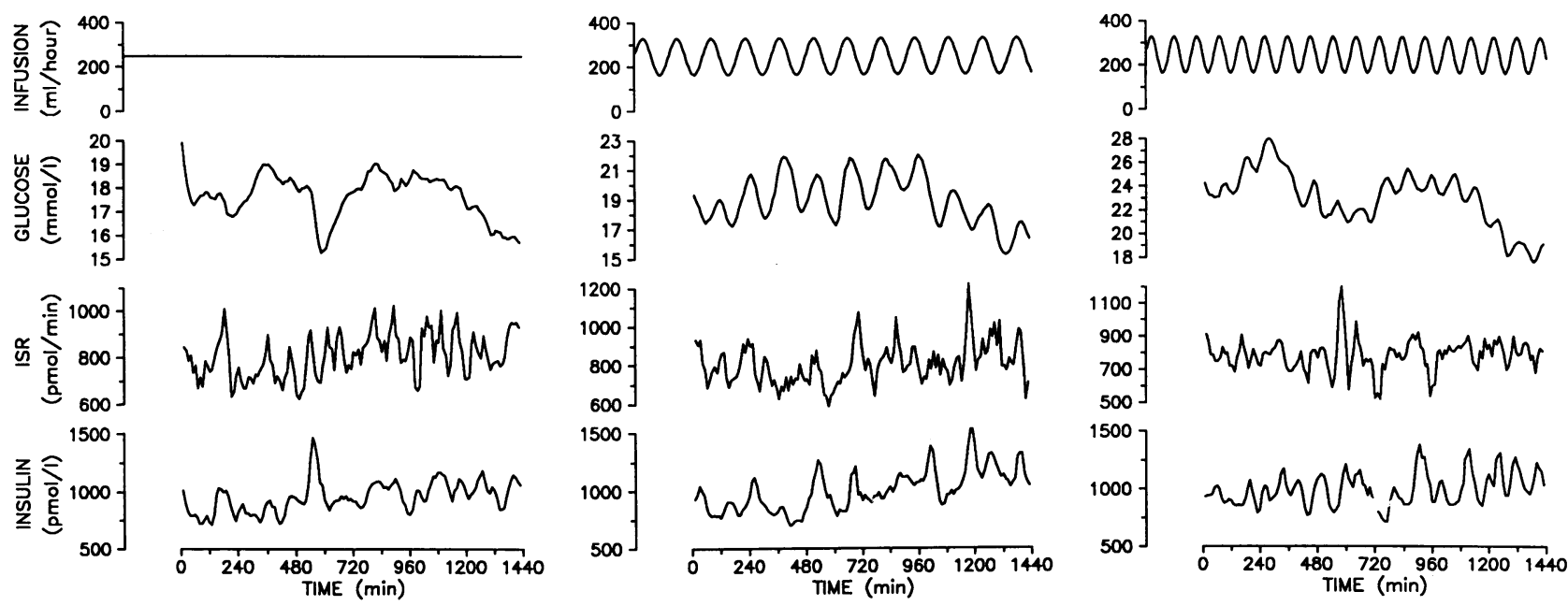

Figure 4. Profiles of the glucose infusion rate, the glucose and insulin concentrations, and the insulin secretory rates in one representative NIDDM patient. All three studies performed in this patient are shown: constant glucose infusion, left; slow oscillatory infusion, middle; rapid oscillatory infusion, right. A few interruptions occurred that were all of short duration $(<2 \mathrm{~min})$, and they did not have any visible effect upon the glucose profiles. 


\begin{tabular}{|c|c|c|c|c|c|c|c|c|}
\hline \multirow[b]{2}{*}{ Study } & \multirow[b]{2}{*}{ Group } & \multicolumn{4}{|c|}{ Glucose } & \multicolumn{3}{|c|}{ ISR } \\
\hline & & $\begin{array}{l}\text { No. of } \\
\text { pulses }\end{array}$ & $\begin{array}{l}\text { Absolute } \\
\text { increment }\end{array}$ & $\begin{array}{l}\% \text { of glucose } \\
\text { pulses with } \\
\text { ISR pulses }\end{array}$ & $\begin{array}{c}\text { No. of } \\
\text { shoulders }\end{array}$ & $\begin{array}{l}\text { No. of } \\
\text { pulses }\end{array}$ & $\begin{array}{l}\text { Absolute } \\
\text { increment }\end{array}$ & $\begin{array}{c}\% \text { of ISR } \\
\text { pulses with } \\
\text { glucose pulses }\end{array}$ \\
\hline \multirow[t]{3}{*}{ Constant glucose infusion } & Control $(n=5)$ & $9.8 \pm 0.6$ & $1.10 \pm 0.12$ & $61.2 \pm 7.9$ & $3.8 \pm 0.4$ & $14.6 \pm 1.0$ & $334 \pm 52$ & $40.4 \pm 3.8$ \\
\hline & IGT $(n=4)$ & $8.3 \pm 0.9$ & $1.18 \pm 0.30$ & $61.3 \pm 9.5$ & $3.5 \pm 1.5$ & $13.5 \pm 1.0$ & $260 \pm 68$ & $39.1 \pm 9.9$ \\
\hline & $\operatorname{NIDDM}(n=7)$ & $6.7 \pm 0.6$ & $1.14 \pm 0.11$ & $54.4 \pm 9.5$ & $6.0 \pm 0.7$ & $12.9 \pm 1.2$ & $158 \pm 17$ & $28.1 \pm 6.1$ \\
\hline \multirow{3}{*}{$\begin{array}{l}\text { Oscillatory glucose infusion } \\
\text { (144 min) }\end{array}$} & Control $(n=5)$ & $10.2 \pm 0.2$ & $3.43 \pm 0.26$ & $70.5 \pm 4.5$ & - & $11.0 \pm 0.5$ & $566 \pm 91$ & $66.4 \pm 2.8$ \\
\hline & $\operatorname{IGT}(n=3)$ & $10.0 \pm 0.0$ & $3.04 \pm 0.23$ & $63.3 \pm 16.7$ & - & $14.3 \pm 0.9$ & $248 \pm 30$ & $46.0 \pm 13.2$ \\
\hline & $\operatorname{NIDDM}(n=6)$ & $9.7 \pm 0.2$ & $2.87 \pm 0.33$ & $40.9 \pm 7.2$ & - & $13.8 \pm 0.7$ & $145 \pm 13$ & $28.5 \pm 5.0$ \\
\hline \multirow{3}{*}{$\begin{array}{l}\text { Oscillatory glucose infusion } \\
\text { (96 min) }\end{array}$} & Control $(n=5)$ & $14.2 \pm 0.2$ & $2.47 \pm 0.13$ & $80.4 \pm 4.0$ & - & $14.2 \pm 0.4$ & $476 \pm 47$ & $80.3 \pm 2.7$ \\
\hline & IGT $(n=4)$ & $14.3 \pm 0.3$ & $2.07 \pm 0.20$ & $61.3 \pm 9.5$ & - & $13.0 \pm 1.2$ & $292 \pm 76$ & $66.1 \pm 4.5$ \\
\hline & $\operatorname{NIDDM}(n=5)$ & $13.4 \pm 0.2$ & $1.57 \pm 0.05$ & $45.8 \pm 10.2$ & - & $14.2 \pm 1.0$ & $159 \pm 17$ & $43.6 \pm 10.2$ \\
\hline
\end{tabular}

Results of statistical analyses are in the text.

any discrepancy between insulin and ISR. In these individuals, the largest spectral peak was always observed at the corresponding period of the exogenous glucose infusion. Examples of individual spectra illustrating these results are shown in Fig. 8.

\section{Discussion}

We have previously demonstrated that in normal volunteers (9), ultradian oscillations of insulin secretion are present during constant intravenous glucose infusion and their period

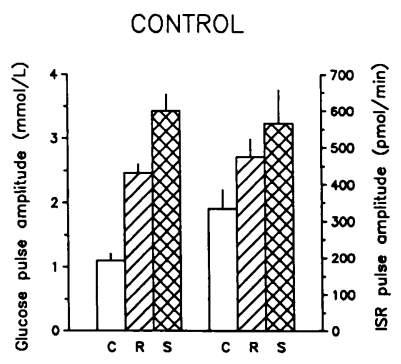

IGT

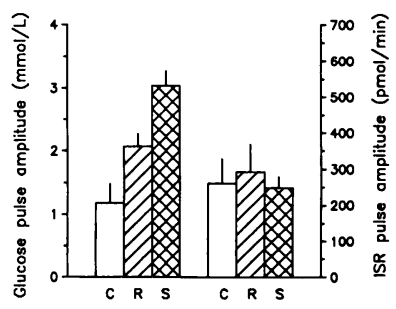

NIDDM

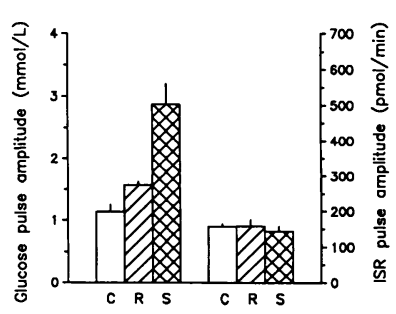

Figure 5. Relationships between absolute amplitudes in the three different groups and the three different protocols. $\mathrm{C}, \mathrm{R}$, and $\mathrm{S}$ denote constant, rapid oscillatory, and slow oscillatory glucose infusion, respectively. In all groups, administration of oscillatory glucose infusion leads to an amplification of the glucose oscillations (lefthand bars), but only in the control group is this associated with a significant increase in the absolute amplitude of the ISR oscillations ( righthand bars). changes to assume the period of exogenous pulses of glucose during exogenous oscillatory glucose infusions. Thus these ultradian oscillations in insulin secretion were completely entrainable by exogenous glucose, implying that the inherent
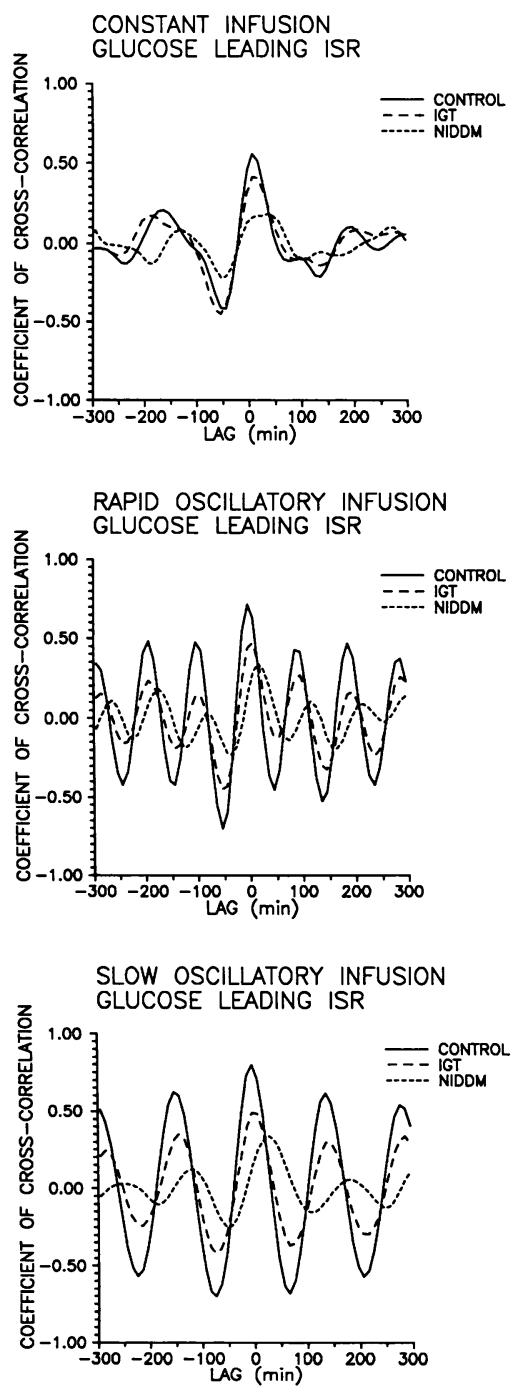

Figure 6. Coefficient of cross-correlation between glucose and ISR in the three study groups during constant (top), rapid oscillatory (center), and slow oscillatory (bottom) glucose infusion. 
CONSTANT

INFUSION

96-MIN

INFUSION

144-MIN

INFUSION
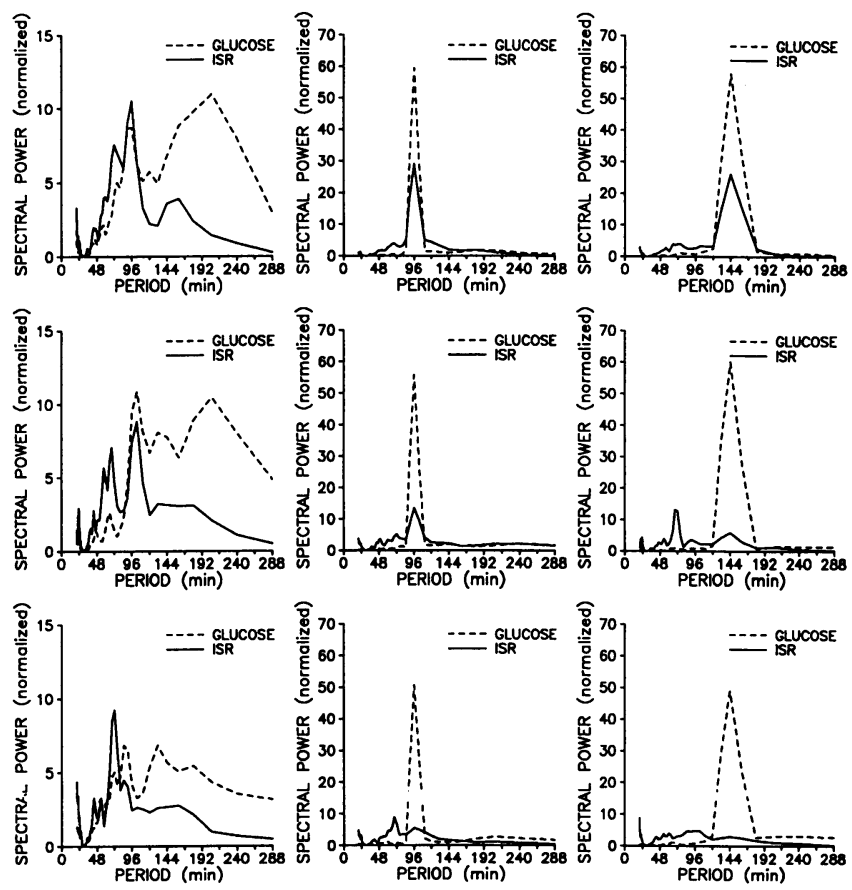

Figure 7. Mean glucose (-- ) and ISR (-) spectra of the control subjects (top), IGT patients (middle) and NIDDM patients (bottom). Error bars have been omitted for clarity. Left, middle, and right columns show the results from the constant, rapid, and slow oscillatory infusion studies, respectively.

properties of the insulin/glucose feedback loop play an important role in regulating and generating the oscillations.

The present study was designed to determine if specific dynamic abnormalities are present in the feedback loop linking insulin secretion and glucose in NIDDM during constant and oscillatory intravenous glucose infusion. Studies were performed in patients with overt NIDDM and matched groups with impaired and normal glucose tolerance who received intravenous glucose infusions either at a constant or oscillatory rate with a periodicity of 96 or $144 \mathrm{~min}$. In all three groups, oscillations in glucose and ISR were apparent during constant glucose infusion. In the group with normal glucose tolerance a tight temporal coupling was evident between the oscillations of glucose and ISR. In contrast, in patients with IGT and NIDDM, there was evidence of uncoupling of this relationship between glucose and ISR and many glucose independent oscillations were apparent. Alterations in the ultradian oscillations in ISR have not previously been characterized in subjects with IGT. The abnormal relationship between oscillations of glucose and ISR in this particular group, which is similar to that observed in the NIDDM group, suggests that an uncoupling of the temporal association between ultradian oscillations in glucose and ISR is an early manifestation of $\beta$-cell dysfunction in NIDDM.

In these studies, evidence for lack of control by glucose of the ultradian oscillations of ISR was demonstrated using three different analytical approaches: (a) by analysis of concomitance of the individual pulses of glucose and ISR using the program ULTRA; $(b)$ by cross-correlation analysis; and $(c)$ by spectral analysis. Analysis of the concomitance of the individual pulses demonstrated that even though the total number of

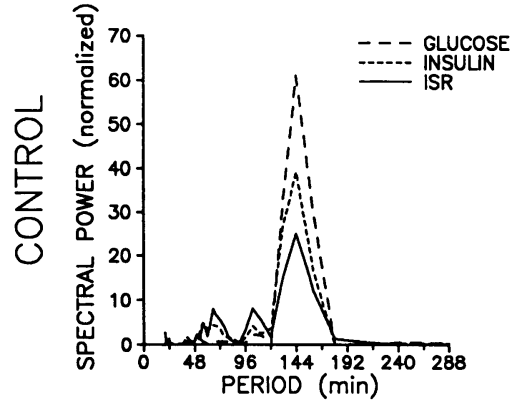

Figure 8. Individual power spectra of the data from one nondiabetic control subject (top), one IGT patient (center), and one NIDDM patient (bottom). The upper two panels correspond to the data shown center of

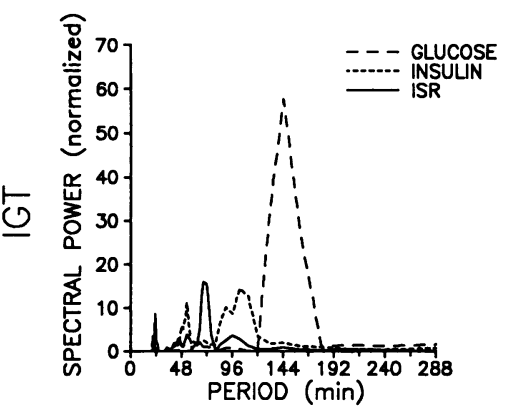

Figs. 2 and 3, while the lower panel is from a diabetic subject different from the one displayed in Fig. 4. In the control subject, glucose $(---)$, insulin (---), and ISR (-) all exhibit a dominant peak at $144 \mathrm{~min}$ (the period of the glucose infusion). In the IGT and NIDDM patients, although

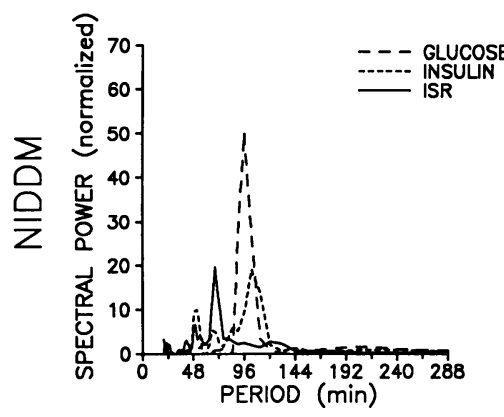
plasma glucose is clearly entrained at $144 \mathrm{~min}$ and $96 \mathrm{~min}$, respectively, this is neither the case with insulin nor with ISR. Furthermore, the dominant period of insulin is different from the dominant period of ISR in both the IGT and NIDDM patients.

glucose and ISR pulses in the 24-h profiles was similar between the groups, a significant reduction in the proportion of pulses in ISR that was concomitant with pulses in glucose and vice versa was observed in NIDDM. Cross-correlation analysis revealed a reduction in the overall concomitance between the ultradian oscillations of glucose, insulin, and ISR, which was significant for both the IGT and NIDDM groups. The differences were most significant when the 144-min oscillatory glucose infusion protocol was used. In addition to reduced coefficients of cross-correlation, this analytical approach also revealed that in the NIDDM group the lag at which the maximal correlation between oscillations of glucose and insulin and between oscillations of glucose and ISR occurred was increased in the NIDDM group. Spectral analysis of the 24-h profiles from the oscillatory glucose infusion studies confirmed the failure of glucose to control the periodicity of the ISR oscillations. In the control subjects, single coinciding peaks representing the period of infusion were clearly apparent in both the glucose and ISR spectra. By contrast, in the subjects with NIDDM and IGT, although a clear peak representing the period of infusion was evident in the glucose spectra, the ISR profiles revealed either a broad spectrum without a clearly dominant period or a dominant period different from the period of infusion and with significantly reduced power in comparison to the controls. We have taken this dissociation between the dominant peaks in the spectra of glucose and ISR to reflect an underlying defect in the 
insulin/glucose feedback mechanism. The results of the spectral analysis go hand in hand with the results shown in Fig. 5, which illustrate that amplification of the glucose oscillations results in a similar amplification of the ISR oscillations in the control group, but not in the IGT and NIDDM groups.

The dissociation between the glucose and ISR oscillations during the oscillatory infusions in the IGT group in this study can thus be added to the list of abnormalities in $\beta$-cell function that have been observed in patients studied in the early stages of NIDDM. Attenuated insulin responses to oral glucose have also been described in subjects with IGT who later develop NIDDM (29-31) and in normoglycemic co-twins of patients with NIDDM (32), a group with a very high risk of developing NIDDM. O'Rahilly and co-workers (16) reported alterations in the rapid oscillations in insulin in first-degree relatives of patients with NIDDM, and Gerich and co-workers (33) observed delayed attenuated responses to oral glucose in patients with mild IGT. The relationship between these abnormalities and the altered ultradian oscillations in the present study is not known nor has the most sensitive test of early $\beta$-cell dysfunction been defined. Nevertheless the data from the current study taken in conjunction with the studies quoted above illustrate that with the appropriate experimental approaches, intrinsic defects in the $\beta$ cell may be demonstrable in patients with impaired glucose tolerance but normal glycosylated hemoglobin values.

The precise mechanisms responsible for the loss of entrainment in the IGT and NIDDM groups are not known. However a model of the interactions between glucose, insulin secretion, and action recently published by our group (12) may provide an initial framework with which to address these questions. In this model, the maintenance of the regular pattern of oscillatory secretion observed in normal subjects was dependent on a number of factors, the most important of these being the simple sigmoidal relationship between glucose and insulin secretion, the existence of a time delay in the order of 25-50 min between the increase in insulin secretion and the suppression of hepatic glucose production, and the fact that insulin must diffuse into a remote peripheral compartment before stimulating peripheral glucose utilization. Alterations in the dose-response relationship between glucose and insulin secretion (34-38), hepatic resistance to the action of insulin (39-43), or prolonged time lags in the periphery due to diminished glucose disposal (42) could all contribute to the alterations in the dynamic interactions between insulin secretion and glucose observed in the IGT and NIDDM groups in this study. To understand fully the lack of entrainment observed in these groups, however, it may be necessary to modify the model to include factors other than the insulin/glucose feedback mechanism.

The present study also highlights the systematic change in $\beta$-cell secretory responses as obesity progresses to IGT and ultimately NIDDM. In Table II, the mean 24-h insulin and C-peptide concentrations as well as the mean insulin secretory rates in the IGT group are intermediate between those observed in the obese and NIDDM groups. The data in Table III demonstrate a progressive decline in the absolute amplitude of the glucose and ISR pulses with the transition from obesity to IGT to NIDDM. The similarity in basal insulin and C-peptide levels between the NIDDM and IGT groups in Table I (both of which were lower than the corresponding concentrations in the obese [nondiabetic] subjects) raises the possibility that $\beta$-cell secretory function decreases before the development of severe hyper- glycemia in NIDDM. This is further illustrated in Fig. 1, depicting the relationship between mean glucose concentration and mean rates of insulin secretion throughout the 24-h study where the insulin secretory responses in many of the IGT and NIDDM subjects were not different despite the marked differences in glucose levels. In this regard, it is also of interest that all three methods used to analyze the interaction between glucose and insulin secretion demonstrated abnormalities in the patients with IGT that were qualitatively similar but less severe than the defects identified in the subjects with NIDDM. It is therefore possible that the uncoupling of the ISR and glucose oscillations is a key factor in the development of glucose intolerance in susceptible individuals.

The lower association observed by both cross-correlation and spectral analysis between the patterns of insulin and ISR oscillations in the IGT and NIDDM groups than in the control group is not readily explained in view of the equimolar secretion of insulin and C-peptide from the $\beta$-cell, which has been well documented (44-46). In contrast to nondiabetic subjects, NIDDM patients have elevated levels of proinsulin and of proinsulin conversion intermediates (split proinsulins), suggesting that the $\beta$-cell in this instance releases immature secretory granules into the circulation (47-52). Since conventional insulin radioimmunoassays cross-react with proinsulin, it is also possible that the dissociation observed between insulin and ISR in the NIDDM and IGT groups can be attributed in part to oscillating proportions of proinsulin secretion in relation to the secretion of insulin. Proinsulin concentrations were not, however, measured in these experiments.

Although $\beta$-cell entrainment was preserved in obesity, the present study raises the possibility that obese subjects may have subtle abnormalities in $\beta$-cell function. Previous studies in obese individuals quantitating insulin secretion rates and evaluating pulsatility following meals have demonstrated $\beta$-cell secretory patterns to be similar to those observed in normal weight subjects $(7,53)$. In the present study, detailed analysis of the proportion of ISR pulses that were concomitant with pulses in glucose yielded concomitance ratios of $66 \%$ and $80 \%$ in the $96 \mathrm{~min}$ and $144 \mathrm{~min}$ oscillatory infusions, respectively. This contrasts with concomitance ratios exceeding $90 \%$ that were previously reported for younger, normal weight subjects (9) studied under identical experimental protocols. The obese subjects in this study were however older and presumably more insulin resistant than the normal weight subjects studied previously. Additional studies are currently under way to determine if these differences could be due to the different ages of the subjects, and/or differences in weight and degree of insulin resistance. In light of the importance of the insulin/glucose feedback mechanism in controlling the oscillations in nondiabetic subjects, the notion that insulin resistance may, together with intrinsic defects in the $\beta$ cell, contribute to alterations in ultradian oscillations is an intriguing possibility that should be systematically addressed in future studies.

In conclusion, the present study demonstrates that the insulin/glucose feedback mechanism, which plays an important role in the regulation and generation of the ultradian oscillations in nondiabetic subjects, is disrupted in NIDDM and even in conditions where glucose tolerance is only minimally impaired. The resultant uncoupling of the normal tight relationship between glucose and ISR pulses is reflected in a reduction in the spectral power of the ultradian oscillations of insulin secretion when oscillatory glucose is administered and in a dis- 
crepancy between the dominant period of the spectra of insulin secretion and glucose. Similar changes are seen using crosscorrelation analysis and pulse analysis. The dissociation between the oscillations becomes more severe with the progression from impaired glucose tolerance to NIDDM. Whether the abnormal secretory oscillations play a pathogenetic role in the progression to overt diabetes or whether they are merely reflective of underlying defects in the $\beta$ cell and peripheral tissues remains to be determined.

\section{Acknowledgments}

The authors wish to thank the nursing staff of the Clinical Research Center for expert care of the subjects who participated in the study. The authors also greatly appreciate the advice given by Theodore Karrison regarding statistical analyses and the technical contributions of Paul Rue and Marsha Jackson.

This work was supported by National Institutes of Health grants DK-31842, DK-13941, DK-20595, DK-26678, (Diabetes Research and Training Center, Clinical Nutrition Research Unit), and RR00055 (Clinical Research Center). Dr. O'Meara (390343) and Dr. Sturis (392574) were recipients of Post Doctoral Fellowships from the Juvenile Diabetes Foundation International.

\section{References}

1. Lang, D. A., D. R. Matthews, J. Peto, and R. C. Turner. 1979. Cyclic oscillations of basal plasma glucose and insulin concentrations in human beings. N. Engl. J. Med. 301:1023-1027.

2. Lang, D. A., D. R. Matthews, M. Burnett, G. M. Ward, and R. C. Turner. 1982. Pulsatile, synchronous basal insulin and glucagon secretion in man. Diabetes. 31:22-26.

3. Hansen, B. C., K. C. Jen, S. B. Pek, and R. A. Wolfe. 1982. Rapid oscillations in plasma insulin, glucagon, and glucose in obese and normal weight humans. J. Clin. Endocrinol. \& Metab. 54:785-792.

4. Matthews, D. R., D. A. Lang, M. A. Burnett, and R. C. Turner. 1983. Control of pulsatile insulin secretion in man. Diabetologia. 24:231-237.

5. Simon, C., M. Follenius, and G. Brandenberger. 1987. Postprandial oscillations of plasma glucose, insulin and C-peptide in man. Diabetologia. 30:769-773.

6. Simon, C., G. Brandenberger, and M. Follenius. 1987. Ultradian oscillations of plasma glucose, insulin, and C-peptide in man during continuous enteral nutrition. J. Clin. Endocrinol. \& Metab. 64:669-674.

7. Polonsky, K. S., B. D. Given, and E. Van Cauter. 1988. Twenty-four-hour profiles and pulsatile patterns of insulin secretion in normal and obese subjects. $J$. Clin. Invest. 81:442-448.

8. Shapiro, E. T., H. Tillil, K. S. Polonsky, V. S. Fang, A. H. Rubenstein, and E. Van Cauter. 1988. Oscillations in insulin secretion during constant glucose infusion in normal man: relationship to changes in plasma glucose. J. Clin. Endocrinol. \& Metab. 67:307-314.

9. Sturis, J., E. Van Cauter, J. D. Blackman, and K. S. Polonsky. 1991. Entrainment of pulsatile insulin secretion by oscillatory glucose infusion. J. Clin. Invest. 87:439-445.

10. Stagner, J. I., E. Samols, and G. C. Weir. 1980. Sustained oscillations of insulin, glucagon, and somatostatin from the isolated canine pancreas during exposure to a constant glucose concentration. J. Clin. Invest. 65:939-942.

11. Chou, H. F., and E. Ipp. 1990. Pulsatile insulin secretion in isolated rat islets. Diabetes. 39:112-117.

12. Sturis, J., K. S. Polonsky, E. Mosekilde, and E. Van Cauter. 1991. Computer model for mechanisms underlying ultradian oscillations of insulin and glucose. Am. J. Physiol. 260:E801-E809.

13. Sturis, J., K. S. Polonsky, J. D. Blackman, C. Knudsen, E. Mosekilde, and E. Van Cauter. 1992. Aspects of oscillatory insulin secretion. In Complexity, Chaos, and Biological Evolution. NATO ASI series B. E. Mosekilde and L. Mosekilde, editors. Plenum Press, New York. 75-93.

14. Glass, L., and M. C. Mackey. 1988. From Clocks to Chaos: the Rhythms of Life. Princeton University Press, Princeton. 248 pp.

15. Lang, D. A., D. R. Matthews, M. Burnett, and R. C. Turner. 1981. Brief, irregular oscillations of basal plasma insulin and glucose concentrations in diabetic man. Diabetes. 30:435-439.

16. O'Rahilly, S., R. C. Turner, and D. R. Matthews. 1988. Impaired pulsatile secretion of insulin in relatives of patients with non-insulin-dependent diabetes. N. Engl. J. Med. 318:1225-1230.

17. Polonsky, K. S., B. D. Given, L. J. Hirsch, H. Tillil, E. T. Shapiro, C.
Beebe, B. H. Frank, J. A. Galloway, and E. Van Cauter. 1988. Abnormal patterns of insulin secretion in non-insulin-dependent diabetes mellitus. N. Engl. J. Med. 318:1231-1239.

18. Simon, C., G. Brandenberger, M. Follenius, and J. L. Schlienger. 1991. Alteration in the temporal organisation of insulin secretion in Type 2 (non-insulin-dependent) diabetic patients under continuous enteral nutrition. Diabetologia. 34:435-440.

19. National Diabetes Data Group. 1979. Classification and diagnosis of diabetes mellitus and other categories of glucose intolerance. Diabetes. 28:10391057.

20. Morgan, C. R., and A. Lazarow. 1963. Immunoassay of insulin: two antibody system: plasma insulin levels of normal, subdiabetic and diabetic rats. Diabetes. 12:115-126.

21. Faber, O. K., C. Binder, J. Markussen, L. G. Heding, V. K. Naithani, H. Kuzuya, P. Blix, D. L. Horwitz, and A. H. Rubenstein. 1978. Characterization of seven C-peptide antisera. Diabetes. 27(Suppl. 1):170-177.

22. Matthews, D. R., J. P. Hosker, A. S. Rudenski, B. A. Naylor, D. F. Treacher, and R. C. Turner. 1985. Homeostasis model assessment: insulin resistance and $\beta$-cell function from fasting plasma glucose and insulin concentrations in man. Diabetologia. 28:412-419.

23. Polonsky, K. S., J. Licinio-Paixao, B. D. Given, W. Pugh, P. Rue, J. Galloway, T. Karrison, and B. Frank. 1986. Use of biosynthetic human C-peptide in the measurement of insulin secretion rates in normal volunteers and type $I$ diabetic patients. J. Clin. Invest. 77:98-105.

24. Van Cauter, E., F. Mestrez, J. Sturis, and K. S. Polonsky. 1992. Estimation of insulin secretion rates from C-peptide levels: comparison of individual and standard kinetic parameters for C-peptide clearance. Diabetes. 41:368-377.

25. Van Cauter, E. 1988. Estimating false-positive and false-negative errors in analyses of hormonal pulsatility. Am. J. Physiol. 254:E786-E794.

26. Cleveland, W. 1979. Robust locally weighted regression and smoothing scatterplots. J. Am. Stat. Assoc. 74:829-836.

27. Fisher, R. A. 1958. Statistical Methods for Research Workers. Oliver \& Boyd, Edinburgh. 356 pp.

28. Jenkins, G. M., and D. G. Watts. 1968. Spectral Analysis and Its Applications. Holden Day, San Francisco. 525 pp.

29. Kosaka, K., R. Hagura, and T. Kuzuya. 1977. Insulin responses in equivocal and definite diabetes, with special reference to subjects who had mild glucose intolerance but later developed definite diabetes. Diabetes. 26:944-952.

30. Kadowaki, T., Y. Miyake, R. Hagura, Y. Akanuma, H. Kajinuma, N. Kuzuya, F. Takaku, and K. Kosaka. 1984. Risk factors for worsening to diabetes in subjects with impaired glucose tolerance. Diabetologia. 26:44-49.

31. Efendic, S., R. Luft, and A. Wajngot. 1984. Aspects of the pathogenesis of type 2 diabetes. Endocr. Rev. 5:395-410.

32. Barnett, A. H., A. J. Spiliopoulos, D. A. Pyke, W. A. Stubbs, J. Burrin, and K. G. Alberti. 1981. Metabolic studies in unaffected co-twins of non-insulin-dependent diabetics. Br. Med. J. 282:1656-1658.

33. Mitrakou, A., D. Kelley, M. Mokan, T. Veneman, T. Pangburn, J. Reilly, and J. Gerich. 1992. Role of suppression of glucose production and diminished early insulin release in impaired glucose tolerance. $N$. Engl. J. Med. 326:22-29.

34. Ward, W. K., D. C. Bolgiano, B. McKnight, J. B. Halter, and D. Porte, Jr. 1984. Diminished B cell secretory capacity in patients with noninsulin-dependent diabetes mellitus. J. Clin. Invest. 74:1318-1328.

35. Pfeifer, M. A., J. B. Halter, and D. Porte, Jr. 1981. Insulin secretion in diabetes mellitus. Am. J. Med. 70:579-588.

36. Garvey, W. T., J. M. Olefsky, J. Griffin, R. F. Hamman, and O. G. Kolterman. 1985. The effect of insulin treatment on insulin secretion and insulin action in type II diabetes mellitus. Diabetes. 34:222-234.

37. Ferner, R. E., L. Ashworth, B. Tronier, and K. G. M. M. Alberti. 1986 Effects of short-term hyperglycemia on insulin secretion in normal humans. $\mathrm{Am}$. J. Physiol. 250:E655-E661.

38. Nesher, R., L. Della Casa, Y. Litvin, J. Sinai, G. Del Rio, B. Pevsner, Y. Wax, and E. Cerasi. 1987. Insulin deficiency and insulin resistance in type 2 (non-insulin-dependent) diabetes: quantitative contributions of pancreatic and peripheral responses to glucose homeostasis. Eur. J. Clin. Invest. 17:266-274.

39. Firth, R., P. Bell, and R. Rizza. 1987. Insulin action in non-insulin dependent diabetes mellitus: the relationship between hepatic and extrahepatic insulin resistance and obesity. Metab. Clin. Exp. 36:1091-1095.

40. Glauber, H., P. Wallace, and G. Brechtel. 1987. Effects of fasting on plasma glucose and prolonged tracer measurement of hepatic glucose output in NIDDM. Diabetes. 36:1187-1194.

41. Bogardus, C., S. Lillioja, B. V. Howard, G. Reaven, and D. Mott. 1984. Relationships between insulin secretion, insulin action, and fasting plasma glucose concentration in nondiabetic and noninsulin-dependent diabetic subjects. $J$. Clin. Invest. 74:1238-1246.

42. Kolterman, O. G., R. S. Gray, J. Griffin, P. Burstein, J. Insel, J. A. Scarlett, and J. M. Olefsky. 1981. Receptor and postreceptor defects contribute to the insulin resistance in noninsulin-dependent diabetes mellitus. J. Clin. Invest. 68:957-969.

43. Powrie, J. K., G. D. Smith, T. R. Hennessy, F. Shojaee-Moradie, J. M. Kelly, P. H. Sönksen, and R. H. Jones. 1992. Incomplete suppression of hepatic 
glucose production in non-insulin dependent diabetes mellitus measured with [6,6- $\left.{ }^{2} \mathrm{H}_{2}\right]$ glucose enriched glucose infusion during the hyperinsulinaemic euglycaemic clamps. Eur. J. Clin. Invest. 22:244-253.

44. Rubenstein, A. H., J. L. Clark, F. Melani, and D. F. Steiner. 1969. Secretion of proinsulin, C-peptide by pancreatic B cells and its circulation in blood. Nature (Lond.). 224:697-699.

45. Steiner, D. F. 1978. On the role of proinsulin C-peptide. Diabetes 27(Suppl. 1):145-148.

46. Horwitz, D. L., J. I. Starr, M. E. Mako, W. G. Blackward, and A. H. Rubenstein. 1975. Proinsulin, insulin and C-peptide concentrations in human portal and peripheral blood. J. Clin. Invest. 55:1278-1283.

47. Duckworth, W. C., and A. E. Kitabachi. 1972. Direct measurements of plasma proinsulin in normal and diabetic subjects. Am. J. Med. 53:418-427.

48. Mako, M. E., J. I. Starr, A. H. Rubenstein. 1977. Circulating proinsulin in patients with maturity onset diabetes. Am. J. Med. 63:865-869.

49. Ward, W. K., E. C. LaCava, T. L. Paquette, J. C. Beard, B. J. Wallum, and D. Porte, Jr. 1987. Disproportionate elevation of immunoreactive proinsulin in type 2 (non-insulin-dependent) diabetes mellitus and in experimental insulin resistance. Diabetologia. 30:698-702.

50. Yoshioka, N., T. Kuzuya, A. Matsuda, M. Taniguch, and Y. Iwamoto. 1988. Serum proinsulin levels at fasting and after oral glucose load in patients with type 2 (non-insulin-dependent) diabetes mellitus. Diabetologia. 31:355360 .

51. Temple, R. C., C. A. Carrington, S. D. Luzio, D. R. Owens, A. E. Schneider, W. J. Sobey, and C. N. Hales. 1989. Insulin deficiency in non-insulindependent diabetes. Lancet 1 (8633):293-295.

52. Saad, M. F. S. E. Kahn, R. G. Nelson, D. J. Pettitt, W. C. Knowler, M. W. Schwartz, S. Kowalyk, P. H. Bennett, and D. Porte, Jr. 1990. Disproportionately elevated proinsulin in Pima Indians with noninsulin-dependent diabetes mellitus. J. Clin. Endocrinol. \& Metab. 70:1247-1253.

53. Polonsky, K. S., B. D. Given, L. Hirsch, E. T. Shapiro, H. Tillil, C. Beebe, J. A. Galloway, B. H. Frank, T. Karrison, and E. Van Cauter. 1988. Quantitative study of insulin secretion and clearance in normal and obese subjects. J. Clin. Invest. 81:435-441. 